\title{
REITERATED HOMOGENIZATION OF NONLINEAR MONOTONE OPERATORS
}

\author{
J. L. LIONS* D. LUKKASSEN ${ }^{* *}$ L. E. PERSSON ${ }^{* * *}$ P. WALL ${ }^{* * *}$ \\ (Dedicated to Professor Jaak Peetre on the Occasion of his 65th Birthday)
}

\begin{abstract}
In this paper, the authors study reiterated homogenization of nonlinear equations of the form $-\operatorname{div}\left(a\left(x, x / \varepsilon, x / \epsilon^{2}, D u_{\varepsilon}\right)\right)=f$, where $a$ is periodic in the first two arguments and monotone in the third. It is proved that $u_{\varepsilon}$ converges weakly in $W^{1, p}(\Omega)$ (and even in some multiscale sense), as $\varepsilon \rightarrow 0$ to the solution $u_{0}$ of a limit problem. Moreover, an explicit expression for the limit problem is given. The main results were also stated in [15]. This article presents the complete proofs of these results.
\end{abstract}

Keywords Homogenization, Nonlinear monotone operators, Nonlinear equation

1991 MR Subject Classification 35B27, 35J60, 73B27

Chinese Library Classification O175.25, O175.29, O341 Document Code A

Article ID 0252-9599(2001)01-0001-12

\section{$\S 1$. Introduction}

Let us consider the class of partial differential equations of the form

$$
-\operatorname{div}\left(a_{\varepsilon}\left(x, D u_{\varepsilon}\right)\right)=f \quad \text { on } \Omega, u_{\varepsilon} \in W_{0}^{1, p}(\Omega),
$$

where $\boldsymbol{a}_{\varepsilon}$ is increasingly oscillating as $\varepsilon \rightarrow 0, \Omega$ is an open bounded subset of $R^{n}, 1<p<\infty$, $1 / p+1 / q=1$ and $f \in W^{-1, q}(\Omega)$. The homogenization problem for (1.1) consists of the study of the asymptotic behavior of solutions $u_{\varepsilon}$, as $\varepsilon \rightarrow 0$. In many important cases $u_{\varepsilon}$ converges weakly in $W_{0}^{1, p}(\Omega)$ to the solution $u_{0}$ of the homogenized problem

$$
-\operatorname{div}\left(b\left(D u_{0}\right)\right)=f \quad \text { on } \Omega, u_{0} \in W_{0}^{1, p}(\Omega) .
$$

The first homogenization result of this kind was given by De Giorgi and Spagnolo in [13] for the linear case when $a_{\varepsilon}$ is of the form $a_{\varepsilon}(x, \xi)=a(x / \varepsilon, \xi)=A(x / \varepsilon) \xi$, where $A(\cdot)$ is a periodic and bounded matrix. In [8] Chiado Piat and Defranceschi considered the case when $a$ is monotone, continuous and satisfies suitable coerciveness and growth conditions in the second variable. The corresponding variational problems have been studied by many authors in the notion of $\Gamma$-convergence (see e.g. the books $[4,11]$ ). Quite recently a new

\footnotetext{
Manuscript received June 27, 2000.

*Collège de France, 3, rue d'Ulm, 75231 Paris cedex 05, France.

**Narvik Institute of Technology and Norut Technology Ltd., N-8505 Narvik, Norway.

***Department of Mathematics, Luleå University of Technology, S-971 87 Luleå, Sweden.

E-mail: larserik@sm.luth.se
} 
method has been developed, known as two-scale convergence, which is well-suited for the analysis of the convergence questions described above (see e.g. [1, 20]).

When $a_{\varepsilon}(x, \xi)=a\left(x / \varepsilon, x / \varepsilon^{2}, \xi\right)$, where $a$ is periodic in the first two variables, one usually speaks about reiterated homogenization. This concept was introduced by Bensoussan, Lions and Papanicolaou in [3], where it was stated a result for the linear operators which later has been known as the iterated homogenization theorem. This theorem has been an indispensable tool in the construction of structures with extreme effective material properties. Concerning this topic we refer to the collection of classical papers in [7], where the introduction gives a good selection of references (see also [17]). Braides and Lukkassen considered the corresponding $\Gamma$-convergence results for convex reiterated problems in [5] and [16] and later on Müller, and subsequently Braides and Defranceschi considered the non-convex case (see [4, p.215]). These results have recently been used to construct nonlinear materials with surprisingly effective behavior (see e.g. $[5,16,22,24]$ and the references therein). In the linear case reiterated homogenization has been studied by multiscale-convergence, which is a generalization of two-scale convergence (see [2]).

In this paper we study reiterated homogenization for the case when $a_{\varepsilon}$ is of the form

$$
a_{\varepsilon}(x, \xi)=a\left(x, \frac{x}{\varepsilon}, \frac{x}{\varepsilon^{2}}, \xi\right),
$$

where $a$ is assumed to satisfy suitable monotonicity conditions in $\xi$ and to be periodic in the second and third arguments. The results have been presented earlier in [15] without detailed proofs. In this paper we give the detailed proofs.

The paper is organized as follows. We have collected some notations and preliminary results in Section 2. In Section 3 we study the nonlinear reiterated homogenization problem corresponding to (1.2) by using $G$-convergence and in Section 4 by multiscale-convergence, respectively.

\section{§. Notation and Preliminary Results}

Let $Y$ and $Z$ be open bounded rectangles in $R^{n}$. $|E|$ denotes the Lebesgue measure of the set $E \subset R^{n}$ and $(\cdot, \cdot)$ is the Euclidean scalar product on $R^{n}$. Moreover, $c$ will be a constant that may differ from one place to another and $h \in N$. The function $\widetilde{\omega}: R \rightarrow R$ is an arbitrary function which is continuous, increasing and $\widetilde{\omega}(0)=0$. By $W_{\text {per }}^{1, p}(Y)$ we denote the set of all functions $u \in W^{1, p}(Y)$ with mean value zero which have the same trace on opposite faces of $Y, W_{\mathrm{per}}^{1, p}(Z)$ is defined in the corresponding way. Every function $u \in W_{\mathrm{per}}^{1, p}(Y)$ can

be extended by periodicity to a function in $W_{\text {loc }}^{1, p}\left(R^{n}\right)$ (in this paper we will not make any distinction between the original function and its extension). Let $\Omega_{i} \subset Y, i=1, \ldots, N$, be a family of disjoint open sets such that

$$
\left|Y \backslash \bigcup_{i=1}^{N} \Omega_{i}\right|=0 \text { and }\left|\partial \Omega_{i}\right|=0 .
$$

Let us fix a function $a: R^{n} \times R^{n} \times R^{n} \rightarrow R^{n}$ which fulfills the conditions:

(1) $a(\cdot, z, \xi)$ is $Y$-periodic for every $\xi \in R^{n}$ and every $z \in R^{n}$.

(2) $a(y, \cdot, \xi)$ is $Z$-periodic and Lebesgue measurable for every $\xi \in R^{n}$ and every $y \in R^{n}$.

(3) There exists two constants $c_{1}, c_{2}>0$ and two constants $\alpha$ and $\beta$, with $0 \leq \alpha \leq$ $\min \{1, p-1\}$ and $\max \{p, 2\} \leq \beta<\infty$ such that $a$ satisfies the following boundedness, 
continuity and monotonicity assumptions:

$$
\begin{aligned}
& a(y, z, 0)=0 \text { for a.e. } y, z \in R^{n}, \\
& \left|a\left(y, z, \xi_{1}\right)-a\left(y, z, \xi_{2}\right)\right| \leq c_{1}\left(1+\left|\xi_{1}\right|+\left|\xi_{2}\right|\right)^{p-1-\alpha}\left|\xi_{1}-\xi_{2}\right|^{\alpha}, \\
& \left(a\left(y, z, \xi_{1}\right)-a\left(y, z, \xi_{2}\right), \xi_{1}-\xi_{2}\right) \geq c_{2}\left(1+\left|\xi_{1}\right|+\left|\xi_{2}\right|\right)^{p-\beta}\left|\xi_{1}-\xi_{2}\right|^{\beta} .
\end{aligned}
$$

(4) $a$ is of the form $a(y, z, \xi)=\sum_{i=1}^{N} \chi_{\Omega_{i}}(y) a_{i}(y, z, \xi)$ and satisfies a continuity condition of the form

$$
\left|a\left(y_{1}, z, \xi\right)-a\left(y_{2}, z, \xi\right)\right|^{q} \leq \omega\left(\left|y_{1}-y_{2}\right|\right)\left(1+|\xi|^{p}\right)
$$

for $y_{1}, y_{2} \in \Omega_{i}, i=1, \cdots, N$, a.e. $z \in R^{n}$ and every $\xi \in R^{n}$, where $\omega: R \rightarrow R$ is continuous, increasing and $\omega(0)=0$.

By (2.1), (2.2), and (2.3) it follows that

$$
\begin{aligned}
|a(y, z, \xi)| & \leq c\left(1+|\xi|^{p-1}\right) \\
|\xi|^{p} & \leq c(1+(a(y, z, \xi), \xi))
\end{aligned}
$$

hold for $y \in R^{n}$, a.e. $z \in R^{n}$ and every $\xi \in R^{n}$. The proof of (2.5) follows immediately by (2.1) and (2.2). To see (2.6), let $a, b, \eta$ be positive constants. By using Young's inequality,

$$
a b \leq \frac{p}{\beta} \eta^{-\frac{\beta}{p}} a^{\frac{\beta}{p}}+\frac{\beta-p}{\beta} \eta^{\frac{\beta}{\beta-p}} b^{\frac{\beta}{\beta-p}},
$$

(2.1) and (2.3) it follows that

$$
\begin{aligned}
|\xi|^{p} & =(1+|\xi|)^{\frac{p(p-\beta)}{\beta}}|\xi|^{p}(1+|\xi|)^{\frac{p(\beta-p)}{\beta}} \\
& \leq \frac{p}{\beta} \eta^{-\frac{\beta}{p}}(1+|\xi|)^{p-\beta}|\xi|^{\beta}+\frac{\beta-p}{\beta} \eta^{\frac{\beta}{\beta-p}}(1+|\xi|)^{p} \\
& \leq \frac{p}{\beta} \eta^{-\frac{\beta}{p}}(a(y, z, \xi), \xi)+\frac{\beta-p}{\beta} \eta^{\frac{\beta}{\beta-p}} 2^{p-1}\left(1+|\xi|^{p}\right) .
\end{aligned}
$$

Now (2.6) follows by choosing $\eta$ sufficiently small.

If $\Omega$ is an open bounded convex set and $1 \leq p<\infty$ then the Poincare-Wirtinger inequality states that there exists a constant $K>0$ such that

$$
\left\|u-M_{\Omega}(u)\right\|_{L^{p}(\Omega)} \leq K\|D u\|_{L^{p}\left(\Omega, R^{n}\right)}
$$

for every $u \in W^{1, p}(\Omega)$, where $M_{\Omega}(u)$ denotes the mean value of $u$ over $\Omega$. A corollary of this is that $\|D \cdot\|_{L^{p}\left(Y, R^{n}\right)}$ defines an equivalent norm on $W_{\text {per }}^{1, p}(Y)$, i.e. there exists constants $k_{1}, k_{2}>0$ such that

$$
k_{1}\|D \cdot\|_{L^{p}\left(Y, R^{n}\right)} \leq\|\cdot\|_{W_{\mathrm{P} e \mathrm{r}}^{1, p}(Y)} \leq k_{2}\|D \cdot\|_{L^{p}\left(Y, R^{n}\right)} \cdot
$$

For the sake of completeness we now formulate two well-known lemmas. The proof of the first lemma can be found in e.g. [21] and the second lemma is a simple case of compensated compactness of F. Murat (see $[18,19]$ ).

Lemma 2.1. Let $g \in L^{q}\left(Y, R^{n}\right)$ be a function such that $\int_{Y}(g, D v) d x=0$ for every $v \in H_{\mathrm{per}}^{1, p}(Y)$. Then $g$ can be extended by periodicity to an element of $L_{\mathrm{loc}}^{q}\left(R^{n}, R^{n}\right)$, still denoted by $g$, such that $-\operatorname{div} g=0$ in $\mathcal{D}^{\prime}\left(R^{n}\right)$.

Lemma 2.2. Let $1<p<\infty$. Moreover, let $\left(v_{h}\right)$ be a sequence in $L^{q}\left(\Omega, R^{n}\right)$ which converges weakly to $v,\left(-\operatorname{div} v_{h}\right)$ converges strongly to $-\operatorname{div} v$ in $W^{-1, q}(\Omega)$ and let $\left(u_{\boldsymbol{h}}\right)$ be a 
sequence which converges weakly to $u$ in $W^{1, p}(\Omega)$. Then

for every $\phi \in C_{0}^{\infty}(\Omega)$.

$$
\int_{\Omega}\left(v_{h}, D u_{h}\right) \phi d x \rightarrow \int_{\Omega}(v, D u) \phi d x
$$

\section{§3. G-Convergence Results}

Let us now consider the weak formulation of (1.1):

$$
\left\{\begin{array}{l}
\int_{\Omega}\left(a\left(\frac{x}{\varepsilon}, \frac{x}{\varepsilon^{2}}, D u_{\varepsilon}\right), D \phi\right)=\langle f, \phi\rangle \quad \text { for every } \phi \in W_{0}^{1, p}(\Omega), \\
u_{\varepsilon} \in W_{0}^{1, p}(\Omega)
\end{array}\right.
$$

where $f \in W^{-1, q}(\Omega)$. By the Hartman-Stampancchia Theorem ${ }^{[14]}$, there exist a unique solution for each $\varepsilon$. Below we state a $G$-convergence result, which is a generalization of the classical iterated homogenization theorem.

Theorem 3.1. Let a satisfy $(2.1)-(2,4)$. Moreover, let $\left(u_{\varepsilon}\right)$ be the solutions of (3.1). Then

$$
\begin{aligned}
u_{\varepsilon} & \rightarrow u_{0} \text { weakly in } W_{0}^{1, p}(\Omega), \\
a\left(\frac{x}{\varepsilon}, \frac{x}{\varepsilon^{2}}, D u_{\varepsilon}\right) & \rightarrow b\left(D u_{0}\right) \text { weakly in } L^{q}\left(\Omega, R^{n}\right),
\end{aligned}
$$

as $\varepsilon \rightarrow 0$, where $u_{0}$ is the unique solution of

$$
\left\{\begin{array}{l}
\int_{\Omega}\left(b\left(D u_{0}\right), D \phi\right) d x=\langle f, \phi\rangle \quad \text { for every } \phi \in W_{0}^{1, p}(\Omega) \\
u_{0} \in W_{0}^{1, p}(\Omega)
\end{array}\right.
$$

The operator $b: R^{n} \rightarrow R^{n}$ is defined as

$$
b(\xi)=\frac{1}{|Y|} \int_{Y} b_{1}\left(y, \xi+D v^{\xi}(y)\right) d y
$$

where $v^{\xi}$ is the unique solution of the cell-problem

$$
\left\{\begin{array}{l}
\int_{Y}\left(b_{1}\left(y, \xi+D v^{\xi}(y)\right), D \phi\right) d y=0 \quad \text { for every } \phi \in W_{\mathrm{per}}^{1, p}(Y) \\
v^{\xi} \in W_{\mathrm{per}}^{1, p}(Y)
\end{array}\right.
$$

The operator $b_{1}: Y \times R^{n} \rightarrow R^{n}$ is defined as

$$
b_{1}(y, \xi)=\frac{1}{|Z|} \int_{Z} a\left(y, z, \xi+D v^{\xi, y}(z)\right) d z
$$

where $v^{\xi, y}$ is the unique solution of the cell-problem

$$
\left\{\begin{array}{l}
\int_{Z}\left(a\left(y, z, \xi+D v^{\xi, y}(z)\right), D \phi\right) d z=0 \quad \text { for every } \phi \in W_{\text {per }}^{1, p}(Z) \\
v^{\xi} \in W_{\text {per }}^{1, p}(Z)
\end{array}\right.
$$

Proof. Without loss of generality we assume that $Y=Z=(0,1)^{n}$ and that $\varepsilon=1 / h$, $h \in N$ (the solutions $u_{\varepsilon}=u_{1 / h}$ will be denoted by $u_{h}$ ). The sequence of solutions $\left(u_{h}\right)$ is bounded in $W^{1, p}(\Omega)$. Indeed, let $\phi=u_{h}$ in (3.1) then it follows by (2.6) and (3.1) that

$$
\begin{aligned}
\int_{\Omega}\left|D u_{h}\right|^{p} d x & \leq c \int_{\Omega} 1+\left(a\left(h x, h^{2} x, D u_{h}\right), D u_{h}\right) d x \\
& \leq c|\Omega|+\left\|\left.f\right|_{W^{-1, q}(\Omega)}\right\| u_{h} \|_{W^{1, p}(\Omega)} \leq c\left(1+\left\|u_{h}\right\|_{W^{1, p}(\Omega)}\right) .
\end{aligned}
$$

If $\left\|u_{h}\right\|_{W^{1, p}(\Omega)} \leq 1$, it is clear that the sequence of solutions $\left(u_{h}\right)$ is bounded in $W^{1, p}(\Omega)$. So let us assume that $\left\|u_{h}\right\|_{W^{1, p}(\Omega)} \geq 1$, then (3.4) implies that

$$
\int_{\Omega}\left|D u_{h}\right|^{p} d x \leq c|| u_{h} \|_{W^{1, p}(\Omega)} .
$$


Due to the Poincaré inequality the norms $\|D \cdot\|_{L^{p}\left(\Omega, R^{n}\right)}$ and $\|\cdot\|_{W^{1, p}(\Omega)}$ on $W_{0}^{1, p}(\Omega)$ are equivalent. The desired boundedness then follows by taking (3.5) into account. Thus there exists a subsequence, still denoted by $\left(u_{h}\right)$, such that

$$
u_{\boldsymbol{h}} \rightarrow u_{*} \text { weakly in } W_{0}^{1, p}(\Omega) .
$$

Let us now define

$$
\eta_{h}=a\left(h x, h^{2} x, D u_{h}\right) .
$$

By (2.1), (2.2), and (3.5) we have that $\eta_{h}$ is bounded in $L^{q}\left(\Omega, R^{n}\right)$. Indeed

$$
\begin{aligned}
\int_{\Omega}\left|\eta_{h}\right|^{q} d x & =\int_{\Omega}\left|a\left(h x, h^{2} x, D u_{h}\right)\right|^{q} d x \\
& \left.\left.\leq c \int_{\Omega}\left(1+\mid D u_{h}\right) \mid\right)^{q(p-1-\alpha)} \mid D u_{h}\right)\left.\right|^{q \alpha} d x \\
& \left.\leq c \int_{\Omega}\left(1+\mid D u_{h}\right) \mid\right)^{p} d x \leq c
\end{aligned}
$$

where $c$ is a constant independent of $h$. This means that there exists a subsequence, still denoted by $\left(\eta_{h}\right)$, such that

$$
\eta_{h} \rightarrow \eta_{\star} \text { weakly in } L^{q}\left(\Omega, R^{n}\right) .
$$

From our original problem (3.1) we have that

In the limit we get

$$
\left\{\begin{array}{l}
\int_{\Omega}\left(a\left(h x, h^{2} x, D u_{h}\right), D \phi\right) d x=\langle f, \phi\rangle \quad \text { for every } \phi \in W_{0}^{1, p}(\Omega), \\
u_{h} \in W_{0}^{1, p}(\Omega)
\end{array}\right.
$$

Especially this means that

$$
\int_{\Omega}\left(\eta_{*}, D \phi\right) d x=\langle f, \phi\rangle \text { for every } \phi \in W_{0}^{1, p}(\Omega)
$$

$$
\int_{\Omega}\left(\eta_{*}, D \phi\right) d x=\langle f, \phi\rangle \text { for every } \phi \in C_{0}^{\infty}(\Omega) .
$$

If we now could show that

$$
\eta_{*}=b\left(D u_{*}\right) \text { for a.e } x \in \Omega,
$$

then it follows by the uniqueness of the solution of the homogenized problem (3.2) that $u_{*}=u_{0}$.

Fix $\xi$ and let $u_{h}^{\xi}$ be defined as the solution of the auxiliary problem:

$$
\left\{\begin{array}{l}
\int_{Y}\left(a\left(x, h x, \xi+D u_{h}^{\xi}\right), D \phi\right)=0 \\
u_{h}^{\xi} \in W_{\text {per }}^{1, p}(Y)
\end{array} \quad \text { for every } \phi \in W_{\text {per }}^{1, p}(Y),\right.
$$

Then, by using the ideas in [6] and [23], it is possible to show that

$$
\begin{aligned}
u_{h}^{\xi} & \rightarrow u_{0}^{\xi} \text { weakly in } W_{\mathrm{per}}^{1, p}(Y), \\
a\left(x, h x, \xi+D u_{h}^{\xi}\right) & \rightarrow b_{1}\left(x, \xi+D u_{0}^{\xi}\right) \text { weakly in } L^{q}\left(Y, R^{n}\right),
\end{aligned}
$$

as $h \rightarrow \infty$, where $u_{0}^{\xi}$ is the unique solution of

$$
\left\{\begin{array}{l}
\int_{Y}\left(b_{1}\left(x, \xi+D u_{0}^{\xi}\right), D \phi\right) d x=0 \\
u_{0}^{\xi} \in W_{\text {per }}^{1, p}(Y)
\end{array} \quad \text { for every } \phi \in W_{\text {per }}^{1, p}(Y)\right.
$$

Let us now define the function

$$
w_{h}^{\xi}(x)=(\xi, x)+\frac{1}{h} u_{h}^{\xi}(h x)
$$


We note that, by periodicity and the fact that if $\left(v_{h}\right)$ is a sequence of $Y$-periodic functions in $L_{\mathrm{loc}}^{p}\left(R^{n}\right)$ such that $v_{h} \rightarrow v$ weakly in $L^{p}(Y)$ and if $w_{h}$ is defined as $w_{h}(x)=v_{h}(h x)$, then $w_{h} \rightarrow \frac{1}{Y} \int_{Y} v(x) d x$, we have the following convergences:

$$
\begin{aligned}
w_{h}^{\xi} & \rightarrow(\xi, \cdot) \text { weakly in } W^{1, p}(\Omega), \\
D w_{h}^{\xi} & \rightarrow \xi \text { weakly in } L^{p}\left(\Omega, R^{n}\right), \\
a\left(h x, h^{2} x, D w_{h}^{\xi}\right) & \rightarrow \int_{Y} b_{1}\left(x, \xi+D u_{0}^{\xi}\right) d x=b(\xi) \text { weakly in } L^{q}\left(\Omega, R^{n}\right) .
\end{aligned}
$$

By the monotonicity of $a$ we have for a fixed $\xi$ that

$$
\int_{\Omega}\left(a\left(h x, h^{2} x, D u_{h}\right)-a\left(h x, h^{2} x, D w_{h}^{\xi}\right), D u_{h}-D w_{h}^{\xi}\right) \phi d x \geq 0,
$$

for every $\phi \in C_{0}^{\infty}(\Omega), \phi \geq 0$. By (3.1), we have that

$$
\int_{\Omega}\left(a\left(h x, h^{2} x, D u_{h}\right), D \phi\right) d x=\langle f, \phi\rangle \text { for every } \phi \in C_{0}^{\infty}(\Omega),
$$

which implies that

$$
-\operatorname{div}\left(\alpha\left(h x, h^{2} x, D u_{h}\right)\right)=-\operatorname{div} \eta_{h} \rightarrow-\operatorname{div} \eta_{\star} \text { in } H^{-1, q}(\Omega) .
$$

From the definition of $u_{h}^{\xi}$ (see (3.7) and Lemma 2.1 we have that

$$
-\operatorname{div}\left(a\left(h x, h^{2} x, D w_{h}^{\xi}\right)\right)=0 \text { on } \Omega .
$$

The compensated compactness lemma, Lemma 2.2, then implies that we have in the limit

$$
\int_{\Omega}\left(\eta_{*}(x)-b(\xi), D u_{*}(x)-\xi\right) \phi(x) d x \geq 0,
$$

for every $\phi \in C_{0}^{\infty}(\Omega), \phi \geq 0$. Hence, for every $\xi \in R^{n}$ we have that

$$
\left(\eta_{*}(x)-b(\xi), D u_{*}(x)-\xi\right) \geq 0 \text { for a.e. } x \in \Omega .
$$

In particular, if $\left(\xi_{m}\right)$ is a countable dense subset in $R^{n}$, then (3.8) implies that

$$
\left(\eta_{*}(x)-b\left(\xi_{m}\right), D u_{*}(x)-\xi_{m}\right) \geq 0 \text { for a.e. } x \in \Omega .
$$

By the continuity of $b$ (see (3.10)), it follows that

$$
\left(\eta_{*}(x)-b(\xi), D u_{*}(x)-\xi\right) \geq 0 \text { for a.e. } x \in \Omega \text { and for every } \xi \in R^{n} .
$$

Since $b$ is monotone and continuous (see Proposition 4.1), we have that $b$ is maximal monotone and the crucial relation (3.6) follows.

We have now proved the theorem up to a subsequence of $\left(u_{h}\right)$. By the uniqueness of the solution of the homogenized equation it follows that it is true for the whole sequence and the proof is complete.

Remark 3.1. The extension of this result to the case with $m$ scales, i.e. when $a$ is on the form $a\left(x / \varepsilon, x / \varepsilon^{2}, \cdots, x / \varepsilon^{m}, \xi\right)$ is straightforward.

The homogenized operator $b$ in Theorem 3.1 has the following structure properties:

Proposition 3.1. Let $b$ be the homogenized operator defined in Theorem 3.1. Then

(i) $b(\cdot)$ is strictly monotone, more precisely

$$
\left(b\left(\xi_{1}\right)-b\left(\xi_{2}\right), \xi_{1}-\xi_{2}\right) \geq c\left(1+\left|\xi_{1}\right|+\left|\xi_{2}\right|\right)^{p-\beta}\left|\xi_{1}-\xi_{2}\right|^{\beta}
$$

for every $\xi_{1}, \xi_{2} \in R^{n}$. 
(ii) $b(\cdot)$ is Lipschitz continuous, more precisely

$$
\left|b\left(\xi_{1}\right)-b\left(\xi_{2}\right)\right| \leq c\left(1+\left|\xi_{1}\right|+\left|\xi_{2}\right|\right)^{p-1-\gamma}\left|\xi_{1}-\xi_{2}\right|^{\gamma},
$$

for every $\xi_{1}, \xi_{2} \in R^{n}$, where $\gamma=\alpha /(\beta(\beta-\alpha))$.

(iii) $b(0)=0$.

Proof. Without loss of generality we assume that $Y=Z=(0,1)^{n}$ and that $\varepsilon=1 / h$, $h \in N$ (we write the indices as $h$ instead of $1 / h$ ).

(i) Let $\xi_{j} \in R^{n}, j=1,2$ and define for a.e. $y \in R^{n}$,

$$
w_{h}^{\xi_{j}, y}(z)=\left(\xi_{j}, z\right)+\frac{1}{h} v^{\xi_{j}, y}(h z)
$$

By the periodicity of $v^{\xi_{j}, y}$ we have that

$$
\begin{aligned}
w_{h}^{\xi_{j}, y} & \rightarrow\left(\xi_{j}, z\right) \text { weakly in } W^{1, p}(Y), \\
D w_{h}^{\xi_{j}, y} & \rightarrow \xi_{j} \text { weakly in } L^{p}\left(Y, R^{n}\right), \\
a\left(y, h z, D w_{h}^{\xi_{j}, y}(z)\right) & \rightarrow b_{1}\left(y, \xi_{j}\right) \text { weakly in } L^{p}\left(Y, R^{n}\right) .
\end{aligned}
$$

The monotonicity condition (2.3) on $a$ and Hölder's reversed inequality imply that

$$
\begin{aligned}
& \int_{Y}\left(a\left(y, h z, D w_{h}^{\xi_{1}, y}\right)-a\left(y, h z, D w_{h}^{\xi_{2}, y}\right), D w_{h}^{\xi_{1}, y}-D w_{h}^{\xi_{2}, y}\right) \phi(z) d z \\
\geq & c \int_{Y}\left(1+\left|D w_{h}^{\xi_{1}, y}\right|+\left|D w_{h}^{\xi_{2}, y}\right|\right)^{p-\beta}\left|D w_{h}^{\xi_{1}, y}-D w_{h}^{\xi_{2}, y}\right|^{\beta} \phi(z) d z \\
\geq & c\left(\int_{Y}\left(1+\left|D w_{h}^{\xi_{1}, y}\right|^{p}+\left|D w_{h}^{\xi_{2}, y}\right|^{p}\right) \phi(z) d z\right)^{\frac{p-\beta}{p}} \\
& \times\left(\int_{Y}\left|D w_{h}^{\xi_{1}, y}-D w_{h}^{\xi_{2}, y}\right|^{p} \phi(z) d z\right)^{\frac{\beta}{p}}
\end{aligned}
$$

for every $\phi \in C_{0}^{\infty}(Y)$ such that $\phi \geq 0$. We apply $\liminf _{h \rightarrow \infty}$ on both sides of this inequality and obtain

$$
\begin{aligned}
& \int_{Y}\left(b_{1}\left(y, \xi_{1}\right)-b_{1}\left(y, \xi_{2}\right), \xi_{1}-\xi_{2}\right) \phi(z) d z \\
\geq & \liminf _{n \rightarrow \infty} c\left(\int_{Y}\left(1+\left|D w_{h}^{\xi_{1}, y}\right|^{p}+\left|D w_{h}^{\xi_{2}, y}\right|^{p}\right) \phi(z) d z\right)^{\frac{p-\beta}{p}} \\
& \times\left(\int_{Y}\left|D w_{h}^{\xi_{1}, y}-D w_{h}^{\xi_{2}, y}\right|^{p} \phi(z) d z\right)^{\frac{\beta}{p}} \\
\geq & \liminf _{n \rightarrow \infty} c\left(\int_{Y}\left(1+\left|D w_{h}^{\xi_{1}, y}\right|^{p}+\left|D w_{h}^{\xi_{2}, y}\right|^{p}\right) \phi(z) d z\right)^{\frac{p-\beta}{p}} \\
& \times \liminf _{n \rightarrow \infty}\left(\int_{Y}\left|D w_{h}^{\xi_{1}, y}-D w_{h}^{\xi_{2}, y}\right|^{p} \phi(z) d z\right)^{\frac{\beta}{p}} \\
\geq & c\left(\int_{Y}\left(1+\left|\xi_{1}\right|^{p}+\left|\xi_{2}\right|^{p}\right) \phi(z) d z\right)^{\frac{p-\beta}{p}}\left(\int_{Y}\left|\xi_{1}-\xi_{2}\right|^{p} \phi(z) d z\right)^{\frac{\beta}{p}} \\
\geq & c \int_{Y}\left(1+\left|\xi_{1}\right|+\left|\xi_{2}\right|\right)^{p-\beta}\left|\xi_{1}-\xi_{2}\right|^{\beta} \phi(z) d z
\end{aligned}
$$

for every $\phi \in C_{0}^{\infty}(Y)$, where on the left hand side we have used (3.12), (3.13), Lemma 2.1 and Lemma 2.2 and on the right hand side we first used (3.12) and secondly the fact that 
every norm is weakly lower semicontinous and finally Jensen's inequality. This implies that

$$
\left.\left(b_{1}\left(y, \xi_{1}\right)-b_{1}\left(y, \xi_{2}\right), \xi_{1}-\xi_{2}\right)\right) \geq c\left(1+\left|\xi_{1}\right|+\left|\xi_{2}\right|\right)^{p-\beta}\left|\xi_{1}-\xi_{2}\right|^{\beta}
$$

for every $\xi_{1}, \xi_{2} \in R^{n}$. Finally, (3.9) follows by using almost the same arguments with $b_{1}$ replaced with $b$.

(ii) First we note that by (2.9), (3.3) and (2.5) imply that

$$
\begin{aligned}
\int_{Z}\left|\xi+D v^{\xi, y}\right|^{p} d z & \leq c \int_{Z} 1+\left(a\left(y, z, \xi+D v^{\xi, y}\right), \xi+D v^{\xi, y}\right) d z \\
& =c \int_{Z} 1+\left(a\left(y, z, \xi+D v^{\xi, y}\right), \xi\right) d z \\
& \leq c \int_{Z} 1+c\left(1+\left|\xi+D v^{\xi, y}\right|^{p-1}\right)|\xi| d z
\end{aligned}
$$

By using the Young inequality we obtain that

$$
\int_{Z}\left|\xi+D v^{\xi, y}\right|^{p} d z \leq c\left(1+|\xi|^{p}\right) .
$$

The definition of $b_{1},(2.2)$, Hölder's inequality and (3.14) imply that

$$
\begin{aligned}
& \left|b_{1}\left(y, \xi_{1}\right)-b_{1}\left(y, \xi_{2}\right)\right|^{q} \\
\leq & \int_{Z}\left|a\left(y, z, \xi_{1}+D v^{\xi_{1}, y}\right)-a\left(y, z, \xi_{2}+D v^{\xi_{2}, y}\right)\right|^{q} d z \\
\leq & c \int_{Z}\left(1+\left|\xi_{1}+D v^{\xi_{1}, y}\right|+\left|\xi_{2}+D v^{\xi_{2}, y}\right|\right)^{q(p-1-\alpha)} \\
& \times\left|\xi_{1}+D v^{\xi_{1}, y}-\left(\xi_{2}+D v^{\xi_{2}, y}\right)\right|^{q \alpha} d z \\
\leq & c\left(\int_{Z}\left(1+\left|\xi_{1}+D v^{\xi_{1}, y}\right|+\left|\xi_{2}+D v^{\xi_{2}, y}\right|\right)^{p} d z\right)^{\frac{p-1-\alpha}{p-1}} \\
& \times\left(\int_{Z}\left|\xi_{1}+D v^{\xi_{1}, y}-\left(\xi_{2}+D v^{\xi_{2}, y}\right)\right|^{p} d z\right)^{\frac{\alpha}{p-1}} \\
\leq & c\left(1+\left|\xi_{1}\right|+\left|\xi_{2}\right|\right)^{\frac{p(p-1-\alpha)}{p-1}} \\
& \times\left(\int_{Z}\left|\xi_{1}+D v^{\xi_{1}, y}-\left(\xi_{2}+D v^{\xi_{2}, y}\right)\right|^{p} d z\right)^{\frac{\alpha}{p-1}} .
\end{aligned}
$$

The second factor in (3.15) satisfies the following estimate

$$
\int_{Z}\left|\xi_{1}+D v^{\xi_{1}, y}-\left(\xi_{2}+D v^{\xi_{2}, y}\right)\right|^{p} d z \leq c\left(1+\left|\xi_{1}\right|^{p}+\left|\xi_{2}\right|^{p}\right)^{\frac{\beta-\alpha-1}{\beta-\alpha}}\left|\xi_{1}-\xi_{2}\right|^{\frac{p}{\beta-\alpha}}
$$

For a proof of this estimate see Lemma 3.4 in [12]. By taking (3.15) and (3.16) into account we get that

$$
\left|b\left(y, \xi_{1}\right)-b\left(y, \xi_{2}\right)\right| \leq c\left(1+\left|\xi_{1}\right|+\left|\xi_{2}\right|\right)^{p-1-\gamma}\left|\xi_{1}-\xi_{2}\right|^{\gamma}
$$

for every $\xi_{1}, \xi_{2} \in R^{n}$, where $\gamma=\alpha /(\beta-\alpha)$. Finally, (3.10) follows by using almost the same arguments with $b_{1}$ replaced with $b$.

(iii) Condition (2.1) implies that $b_{1}(y, 0)=0$ which in turn implies that $b(0)=0$.

\section{$\S 4$. Multiscale Convergence Results}

It is possible to extend the above result to the case when $a_{\varepsilon}$ is of the form $a_{\varepsilon}(x, \xi)=$ $a\left(x, x / \varepsilon, x / \varepsilon^{2}, \xi\right)$. In this section we assume that $a_{\varepsilon}$ is of this form. 
A sequence $u_{\varepsilon}$ is said to 3 -scale converge to $u_{0}(x, y, z) \in L^{p}(\Omega, Y, Z)$ if

$$
\lim _{\varepsilon \rightarrow \infty} \int_{\Omega} u_{\varepsilon}(x) \varphi\left(x, \frac{x}{\varepsilon}, \frac{x}{\varepsilon^{2}}\right) d x=\frac{1}{|Y||Z|} \int_{\Omega \times Y \times Z} u_{0}(x, y, z) \varphi(x, y, z) d x d y d z
$$

for all $\varphi \in \mathcal{D}\left(\Omega ; C_{\text {per }}^{\infty}(Y, Z)\right)$ (i.e. functions $\varphi(x, y, z)$ which are $C^{\infty}$ with compact support in $x$ with values of functions which are $C^{\infty}$ and periodic in $y$ and $z$ ). This definition is a variant of the one introduced in [2] for the case $p=2$ (see also $[1,9,20]$ ).

Theorem 4.1. Let $u_{\varepsilon}$ be a solution of (1.1). Then as $\varepsilon \rightarrow 0, u_{\varepsilon}$ 3-scale converges to $u_{0} \in W_{0}^{1, p}(\Omega)$ and $D u_{\varepsilon}$ 3-scale converges to $D u_{0}+D_{y} u_{1}+D_{z} u_{2}$, where $\left\{u_{0}, u_{1}, u_{2}\right\}$ is a solution of

$$
\begin{aligned}
& \frac{1}{|Y||Z|} \int_{\Omega \times Y \times Z}\left(a\left(x, y, z, D u_{0}+D_{y} u_{1}+D_{z} u_{2}\right), D v_{0}+D_{y} v_{1}+D_{z} v_{2}\right) d x d y d z \\
= & \int_{\Omega} f v_{0} d x \text { for all } v_{0} \in W_{0}^{1, p}(\Omega), \quad v_{1} \in L^{p}\left(\Omega ; W_{\mathrm{per}}^{1, p}(Y)\right), \quad v_{2} \in L^{p}\left(\Omega ; W_{\mathrm{per}}^{1, p}(Y \times Z)\right) .
\end{aligned}
$$

Remark 4.1. The theorem above can easily be extended to the case when $a_{\varepsilon}$ is on the form

$$
a_{\varepsilon}(x, \xi)=a\left(x, x / \varepsilon, \cdots, x / \varepsilon^{m}, \xi\right) .
$$

Proof. The proof is divided into several steps:

Step 1. $\left\{u_{\varepsilon}\right\}$ is bounded in $W_{0}^{1, p}(\Omega)$, so one can extract a subsequence such that $u_{\varepsilon} \rightarrow u_{0}$ weakly in $W_{0}^{1, p}(\Omega)$.

Step 2. For each bounded sequence $\left\{u_{\varepsilon}\right\}$ in $L^{p}(\Omega)$ there exists a subsequence and $u_{0} \in L^{p}(\Omega, Y, Z)$ such that $u_{\varepsilon} 3$-scale converges to $u_{0}$ (this is a theorem of Nguetseng for $p=2$ and two scales). The proof is as follows: Observe that

$$
\left|\int_{\Omega} u_{\varepsilon}(x) \varphi\left(x, \frac{x}{\varepsilon}, \frac{x}{\varepsilon^{2}}\right) d x\right| \leq\left\|u_{\varepsilon}\right\|_{L^{p}(\Omega)}\left\|\varphi\left(x, \frac{x}{\varepsilon}, \frac{x}{\varepsilon^{2}}\right)\right\|_{L^{q}(\Omega)} .
$$

In the two-scale case it is possible to prove that for any $\psi \in L^{2}\left(\Omega ; C_{\text {per }}^{\infty}(Y)\right)$ it holds that

$$
\int_{\Omega}\left|\psi\left(x, \frac{x}{\varepsilon}\right)\right|^{2} d x \rightarrow \frac{1}{|Y|} \int_{\Omega \times Y}|\psi(x, y)|^{2} d x d y
$$

as $\varepsilon \rightarrow 0$ (see e.g. Lemma 5.3 in [10]). This result is easily extendable to 3 scales. Thus we obtain that

$$
\begin{aligned}
\int_{\Omega}\left(\left|\varphi\left(x, \frac{x}{\varepsilon}, \frac{x}{\varepsilon^{2}}\right)\right|^{\frac{q}{2}}\right)^{2} d x & \rightarrow \frac{1}{|Y||Z|} \int_{\Omega \times Y \times Z}|\varphi(x, y, z)|^{q} d x d y d z \\
& =\frac{1}{|Y||Z|}\|\varphi\|_{L^{q}(\Omega \times Y \times Z)}^{q} .
\end{aligned}
$$

Accordingly, we obtain that there exists a constant $c$ which is independent of $\varphi$ and a subsequence of $\{\varepsilon\}$ (which may depend on $\varphi$ ) such that

$$
\left|\int_{\Omega} u_{\varepsilon}(x) \varphi\left(x, \frac{x}{\varepsilon}, \frac{x}{\varepsilon^{2}}\right) d x\right| \leq c\|\varphi\|_{L^{q}(\Omega \times Y \times Z)} .
$$

By choosing a countable subset of $\mathcal{D}\left(\Omega ; C_{\text {per }}^{\infty}(Y, Z)\right)$ which is dense in $L^{q}(\Omega, Y, Z)$ and using a standard diagonalization process, we can extract a subsequence of $\{\varepsilon\}$ such that (4.3) holds for all $\varphi$ in this subset with a constant $c$ which is independent of $\varphi$ and $\varepsilon$. It is possible to show that $\mathcal{D}\left(\Omega ; C_{\text {per }}^{\infty}(Y, Z)\right)$ is dense in $L^{q}(\Omega, Y, Z)$ so

$$
F_{\varepsilon}(\varphi)=\int_{\Omega} u_{\varepsilon}(x) \varphi\left(x, \frac{x}{\varepsilon}, \frac{x}{\varepsilon^{2}}\right) d x
$$


defines a bounded sequence $\left\{F_{\varepsilon}\right\}$ of linear functionals on $L^{q}(\Omega, Y, Z)$. Since $L^{p}(\Omega, Y, Z)$ is separable, there exists a subsequence of $\{\varepsilon\}$ and a bounded linear functional $F_{0}$ such that

$$
F_{\varepsilon}(\varphi) \rightarrow F_{0}(\varphi) \text { for all } \varphi \in L^{q}(\Omega, Y, Z) .
$$

Finally, Riesz Representation Theorem gives the existence of a function $u_{0}$ such that (4.1) is satisfied.

Step 3. Let $u_{\varepsilon} \in L^{p}(\Omega)$ and suppose $u_{\varepsilon} 3$-scale converges to $u_{0}$. Then

$$
u_{\varepsilon} \rightarrow \frac{1}{|Y||Z|} \int_{Y \times Z} u_{0}(x, y, z) d y d z
$$

weakly in $L^{p}(\Omega)$. This is seen by taking in (4.1) test functions $\varphi$, which depends only on $x$.

Step 4. Let $u_{\varepsilon} \rightarrow u_{0}$ weakly in $W_{0}^{1, p}(\Omega)$. Then

(i) $u_{\varepsilon} 3$-scale converges to $u_{0}$,

(ii) There exist $u_{1} \in L^{p}\left(\Omega ; W_{\text {per }}^{1, p}(Y)\right)$ and $u_{2} \in L^{p}\left(\Omega ; W_{\text {per }}^{1, p}(Y \times Z)\right)$ such that $D u_{\varepsilon}$ 3-scale converges to $D u_{0}+D_{y} u_{1}+D_{z} u_{2}$.

Proof. By Step 2 (extension of Step 2 to vectors is immediate) there exist $\tilde{u}_{0}(x, y, z)$ and $\chi_{0}(x, y, z)$ such that, up to a subsequence, $u_{\varepsilon}$ and $D u_{\varepsilon} 3$-scale converge to $\tilde{u}_{0}$ and $\chi_{0}$, respectively. Using Step 2 in the identity

$$
\varepsilon^{2} \int_{\Omega}\left(D u_{\varepsilon}\right) \Phi\left(x, \frac{x}{\varepsilon}, \frac{x}{\varepsilon^{2}}\right) d x=-\int_{\Omega} u_{\varepsilon}\left(\varepsilon^{2} \operatorname{div}_{x} \Phi+\varepsilon \operatorname{div}_{y} \Phi+\operatorname{div}_{z} \Phi\right) d x,
$$

we obtain in the limit that

$$
0=-\frac{1}{|Y||Z|} \int_{\Omega \times Y \times Z}\left(u_{0} \operatorname{div}_{z} \Phi\right) d x d y d z .
$$

Thus $u_{0}$ is independent of $z$. Letting $\Phi$ be independent of the third variable we obtain the identity

$$
\varepsilon \int_{\Omega}\left(D u_{\varepsilon}\right) \Phi\left(x, \frac{x}{\varepsilon}\right) d x=-\int_{\Omega} u_{\varepsilon}\left(\varepsilon \operatorname{div}_{x} \Phi+\operatorname{div}_{y} \Phi\right) d x,
$$

which in the limit yields the identity

$$
0=-\frac{1}{|Y|} \int_{\Omega \times Y}\left(u_{0} \operatorname{div}_{y} \Phi\right) d x d y
$$

Thus $u_{0}$ is independent of $y$. Next we choose $\Phi(x, y, z)$ such that

$$
\operatorname{div}_{y} \Phi=0, \quad \operatorname{div}_{z} \Phi=0 .
$$

Then, the identity

$$
\int_{\Omega} u_{\varepsilon} \operatorname{div}_{x} \Phi d x=-\int_{\Omega}\left(D u_{\varepsilon}\right) \Phi\left(x, \frac{x}{\varepsilon}, \frac{x}{\varepsilon^{2}}\right) d x
$$

gives

$$
\frac{1}{|Y||Z|} \int_{\Omega \times Y \times Z} u_{0} \operatorname{div}_{x} \Phi d x d y d z=-\frac{1}{|Y||Z|} \int_{\Omega \times Y \times Z} \chi_{0} \Phi(x, y, z) d x d y d z .
$$

Hence we have the existence of $u_{1}$ and $u_{2}$ such that

$$
\chi_{0}=D u_{0}+D_{y} u_{1}+D_{z} u_{2} .
$$

This is seen by using the fact that the orthogonal of divergence-free functions are exactly the gradients. 
Step 5. Let $w_{\varepsilon}$ be defined as

$$
w_{\varepsilon}=w_{0}(x)+\varepsilon w_{1}\left(x, \frac{x}{\varepsilon}\right)+\varepsilon^{2} w_{2}\left(x, \frac{x}{\varepsilon}, \frac{x}{\varepsilon^{2}}\right) .
$$

Then the monotonicity assumption on $a$ implies that

$$
\int_{\Omega}\left(a\left(x, \frac{x}{\varepsilon}, \frac{x}{\varepsilon^{2}}, D u_{\varepsilon}\right)-a\left(x, \frac{x}{\varepsilon}, \frac{x}{\varepsilon^{2}}, D w_{\varepsilon}\right), D\left(u_{\varepsilon}-w_{\varepsilon}\right)\right) d x \geq 0,
$$

i.e.

$$
\int_{\Omega} f\left(u_{\varepsilon}-w_{\varepsilon}\right) d x-\int_{\Omega}\left(a\left(x, \frac{x}{\varepsilon}, \frac{x}{\varepsilon^{2}}, D w_{\varepsilon}\right), D\left(u_{\varepsilon}-D w_{\varepsilon}\right)\right) d x \geq 0 .
$$

Since $w_{0}(x), w_{1}(x, y), w_{2}(x, y, z)$ are given, one can pass to the limit in (4.4) and we obtain that

$$
\begin{aligned}
& \int_{\Omega} f\left(u_{0}-w_{0}\right) d x-\frac{1}{|Y||Z|} \int_{\Omega \times Y \times Z}\left(a \left(x, y, z, D w_{0}+D_{y} w_{1}\right.\right. \\
& \left.\left.+D_{z} w_{2}\right), D\left(u_{0}-w_{0}\right)+D_{y}\left(u_{1}-w_{1}\right)+D_{z}\left(u_{2}-w_{2}\right)\right) d x d y d z \geq 0 .
\end{aligned}
$$

We use then the standard trick of monotonicity, we take in (4.5)

$$
w_{i}=u_{i}-\lambda v_{i}, \lambda>0,
$$

where $v_{0}=v_{0}(x), v_{1}=v_{1}(x, y)$ and $v_{2}=v_{2}(x, y, z)$. After dividing by $\lambda$ we obtain that

$$
\begin{aligned}
& \int_{\Omega} f v_{0} d x-\frac{1}{|Y||Z|} \int_{\Omega \times Y \times Z}\left(a \left(x, y, z, D u_{0}+D_{y} u_{1}+D_{z} u_{2}\right.\right. \\
& \left.\left.-\lambda\left(D v_{0}+D_{y} v_{1}+D_{z} v_{2}\right)\right), D v_{0}+D_{y} v_{1}+D_{z} v_{2}\right) d x d y d z \geq 0 .
\end{aligned}
$$

By letting $\lambda \rightarrow 0$ we obtain that

$$
\int_{\Omega} f v_{0} d x=\frac{1}{|Y||Z|} \int_{\Omega \times Y \times Z}\left(a\left(x, y, z, D u_{0}+D_{y} u_{1}+D_{z} u_{2}\right), D v_{0}+D_{y} v_{1}+D_{z} v_{2}\right) d x d y d z
$$

and the proof is completed.

\section{REFERENCES}

[1] Allaire, G., Homogenization and two-scale convergence [J], SIAM J. Math. Anal., 23:6(1992), 14821518.

[2] Allaire, G. \& Briane, M., Multiscale convergence and reiterated homogenization [J], Proceedings of the Royal Society of Edinburgh, 126 A(1996), 297-342.

[3] Bensoussan, A., Lions, J. L. \& Papanicolaou, G. C., Asymptotic analysis for periodic structures [M], North Holland, Amsterdam, 1978.

[4] Braides, A. \& Defranceschi, A., Homogenization of multiple integrals [M], Oxford Science Publications, Oxford, 1998.

[5] Braides, a. \& Lukkassen, D., Reiterated homogenization of integral functionals [J], Math. Mod. Meth. Appl. Sci., 10:1(2000), 47-71.

[6] Byström, J., Correctors for some nonlinear monotone operators [J], J. Nonlin. Math. Physics (to appear), (2000).

[7] Cherkaev, A. \& Kohn, R., editors, Topics in the mathematical modelling of composite materials [C], Birkhäuser, Boston, 1997.

[8] Chiado Piat, V. \& Defranceschi, A., Homogenization of monotone operators [J], Nonlinear Analysis, Theory, Methods and Applications, 14:9(1990), 717-732.

[9] Cioranescu, D. \& Donato, P., Introduction to homogenization [M], Oxford University Press, Oxford, 1999.

[10] Conca, C., Planchard, J. \& Vanninathan, M., Fluids and periodic structures [M], Wiley, 1995.

[11] Dal Maso, G., An introduction to $\Gamma$-convergence [M], Birkhäuser, Boston, 1993.

[12] Dal Maso, G. \& Defranceschi, A., Correctors for the homogenization of monotone operators [J], Differential and Integral Equations, 3:6(1990), 1151-1166. 
[13] De Giorgi, E. \& Spagnolo, S., Sulla convergenza degli intehrali dell'energia per operaori ellittici del secondo ordine [J], Boll. Un. Mat. Ital., 8(1973), 391-411.

[14] Hartman, P. \& Stampacchia, G., On some nonlinear elliptic differential functional equations [J], Acta Math., 115(1966), 153-188.

[15] Lions, J. L., Lukkassen, D., Persson, L. E. \& Wall, P., Reiterated homogenization of monotone operators $[\mathrm{J}]$, C. R. Acad. Sci. Series I, 330:8(2000), 675-680.

[16] Lukkassen, D., Formulae and bounds connected to optimal design and homogenization of partial differential operators and integral functionals [D], Ph.D. thesis, Dept. of Math., Tromsö University, Norway, 1996.

[17] Lukkassen, D., A new reiterated structure with optimal macroscopic behaviour [J], SIAM J. Appl. Math., 59:5(1999), 1825-1842.

[18] Murat, F., Compacité parcompensation [J], Ann. Sc. Norm. Sup. Pisa Cl. Sci., 5:4(1978), 489-507.

[19] Murat, F., Compacité parcompensation II [A], Proc. Int. Meeting on "Recent Methods in Nonlinear Analysis" [C], Rome 1978, ed. E. De Giorgi, E. Magenes, U. Mosco, Pitagora ed Bologna, 1979, 245-256.

[20] Nguetseng, G., A general convergence result for a functional related to the theory of homogenization [J], SIAM J. Math. Anal., 20:3(1989), 608-623.

[21] Suquet, P., Plasticité et Homogénéisation [D], Ph.D. thesis, Univ. Paris VI, 1982.

[22] Wall, P., Homogenization of some partial differential operators and integral functionals [D], Ph.D. thesis, Dept. of Math., Luleå University of Technology, Luleå, Sweden, 1998.

[23] Wall, P., Some homogenization and corrector results for nonlinear monotone operators [J], J. Nonlin. Math. Physics, 5(1998), 331-348.

[24] Wall, P., Optimal bounds on the effective shear moduli for some nonlinear and reiterated problems [J], Acta. Sci. Math., 65(2000), 553-566. 